\title{
The Modified Tolman-Oppenheimer-Volkov (TOV) Equation and the Effect of Charge on Pressure in Charge Anisotropy
}

\author{
Petarpa Boonserm ${ }^{1,}$,, Napasorn Jongjittanon², Tritos Ngampitipan ${ }^{3}$ \\ ${ }^{1}$ Department of Mathematics and Computer Science, Faculty of Science, Chulalongkorn University, Bangkok, Thailand \\ ${ }^{2}$ Department of Physics, Faculty of Science, Chulalongkorn University, Bangkok, Thailand \\ ${ }^{3}$ Faculty of Science, Chandrakasem Rajabhat University, Bangkok, Thailand
}

\section{Email address:}

Petarpa.Boonserm@gmail.com (P. Boonserm),njongjittanon@gmail.com (N. Jongjittanon), tritos.ngampitipan@gmail.com (T. Ngampitipan) ${ }^{*}$ Corresponding author

\section{To cite this article:}

Petarpa Boonserm, Napasorn Jongjittanon, Tritos Ngampitipan. The Modified Tolman-Oppenheimer-Volkov (TOV) Equation and the Effect of Charge on Pressure in Charge Anisotropy. American Journal of Physics and Applications. Vol. 4, No. 2, 2016, pp. 57-63.

doi: 10.11648/j.ajpa.20160402.14

Received: February 28, 2016; Accepted: March 18, 2016; Published: March 25, 2016

\begin{abstract}
The Tolman-Oppenheimer-Volkov (TOV) equation describes the interior properties of spherical static perfect fluid object as a relationship between two physical observables - pressure and density. For a fluid sphere object, which contains electric charge, magnetic field, and scalar field, the pressure becomes anisotropic. In the previous article [Phys. Rev. D 76 (2007) 044024; gr-qc/0607001], we deformed TOV in terms of $\delta \rho_{c}$ and $\delta p_{c}$, and we found a new physical and mathematical interpretation for the TOV equation. In this work, we cannot use the perfect fluid constrains because of the electromagnetic field and the massless scalar field within this object. The TOV equation was thus generalized to involve the electromagnetic and the scalar fields. This model is close to the realistic objects in our universe such as a neutron star. In this paper, we consider the modified TOV equation for Schwarzschild coordinates in a special case. The density is considered as a constant and the scalar field is considered absent. On the general model of the TOV equation, the pressure is expressed in terms of radius. However, this model shows that pressure is affected by electric charge. Moreover, we also calculate the rigorous bound on the transmission probability for the Tolman-Bayin type of charged fluid sphere.
\end{abstract}

Keywords: General Relativity, Modified TOV Equation, Charge Anisotropy, Transmission Probability

\section{Introduction}

Several general relativistic isotropic stars can be modeled by perfect fluid spheres [1-2]. A perfect fluid is one with no viscosity, shear stresses, or heat conduction. It is one of the exact solutions to the Einstein's field equation, which is the core equation of general relativity established by Einstein in 1915. A perfect fluid, as a solution to the Einstein's field equation, interests physicists because the equation seems impossible to be solved exactly. Therefore, solving for a perfect fluid poses a great challenge. Several perfect fluid sphere solutions have been generated in literature by directly solving Einstein's equation. These known solutions can also, alternatively, be generated by algorithmic techniques [2]. One of the algorithmic techniques is the solution generating theorem [3]. These generating theorems can produce new solutions for perfect fluid spheres without directly solving the Einstein's field equation. Sometimes, already known solutions are recovered. Sometimes, new (previously unknown) solutions are obtained. Moreover, these theorems can also be used as criteria to classify metrics into seed and non-seed metrics [3]. Furthermore, by rewriting theses theorems in terms of pressure and density, we obtain new solutions for the Tolman-Oppenheimer-Volkov (TOV) equation [4]. When charges are added into perfect fluid spheres, new features arise. These charges make the fluid spheres anisotropic. Anisotropic spheres can be used as models for many charged stars [10]. Moreover, the charges can change the pressure and density profile of stars [4]. The scalar field can also make fluid spheres anisotropic [5]. With charge and scalar field, a generalized TOV equation is formed [10]. In this project, generating theorems will be developed for anisotropic spheres. Following 
development, the generating theorems will be applied to some known anisotropic spheres. The solution generating theorems for perfect fluid spheres can be found in reference [3]. These theorems facilitate us in terms of not having to solve the Einstein's field equation directly to obtain new solutions.

For the solution generating theorems, the theorem transformations are based on spacetime geometry. But as for physical measurements, an object can more easily be observed in terms of mass and energy rather than the geometry of spacetime around it. Because the object's matter can be expressed by energy-momentum-stress tensor in terms of pressure and density, the solution generating theorems should be applied on the physical observables: pressure and density. In addition, this paper is based on general relativity. One of the exact solutions to Einstein's equation is a perfect fluid sphere. Perfect fluid spheres can be made anisotropic by the existence of charge and scalar field. This consequently results in a more complicated form of the Einstein's equation, which is evidently more difficult to solve.

The relationship between the pressure and density profile is given in the Tolman-Oppenheimer-Volkov [TOV] equation [4];

$$
\begin{gathered}
\frac{d p(r)}{d r}=-\frac{[\rho(r)+p(r)]\left[m(r)+4 \pi p(r) r^{3}\right]}{r^{2}\left[1-\frac{2 m(r)}{r}\right]}, \\
\frac{d m(r)}{d r}=4 \pi \rho(r) r^{2} .
\end{gathered}
$$

These equations are inertia solution of static perfect fluid sphere objects, which are derived from the Schwarzschild metric. For the two physical observables, it is also easy to check for physical reasonableness of the quantities. Assuming that we obtain the pressure $p_{0}$ and the density $\rho_{0}$, we can transform them to new solutions in terms of $p+\delta p$ and $\rho+\delta \rho$, and take the TOV equation as a Riccati equation.

Referring to the article by P. Boonserm et al. [4], the solution generating theorems for the TOV equation has been developed as follows.

Theorem (P1) [4]. Let $p_{0}(r)$ and $\rho_{0}(r)$ solve the TOV equation, and hold $m_{0}(r)=4 \pi \int \rho_{0}(r) r^{2} d r$ as fixed. Define an auxiliary function $g_{0}(r)$ by

$$
g_{0}=\frac{m_{0}(r)+4 \pi p_{0}(r) r^{3}}{r^{2}\left[1-2 m_{0}(r) / r\right]} .
$$

Then the general solution to the TOV equation is $p(r)=$ $p_{0}(r)+\delta p(r)$ where

$$
\delta p(r)=\frac{\delta p_{c} \sqrt{1-2 m_{0} / r} \exp \left\{-2 \int_{0}^{r} g_{0} d r\right\}}{1+4 \pi \delta p_{c} \int_{0}^{r} \frac{1}{\sqrt{1-2 m_{0} / r}} \exp \left\{-2 \int_{0}^{r} g_{0} d r\right\} r d r}
$$

where $\delta p_{c}$ is the shift in the central pressure.

Theorem (P2) [4]. Let $p_{0}(r)$ and $\rho_{0}(r)$ solve the TOV equation, and hold $g_{0}$ fixed, such that

$$
g_{0}=\frac{m_{0}(r)+4 \pi p_{0}(r) r^{3}}{r^{2}\left[1-2 m_{0}(r) / r\right]}=\frac{m(r)+4 \pi p(r) r^{3}}{r^{2}[1-2 m(r) / r]}
$$

Then the general solution to the TOV equation is given by $p(r)=p_{0}(r)+\delta p(r)$ and $m(r)=m_{0}(r)+\delta m(r)$ where

$$
\delta m(r)=\frac{4 \pi r^{3} \delta \rho_{c}}{3\left[1+r g_{0}\right]^{2}} \exp \left\{2 \int g_{0} \frac{1-r g_{0}}{1+r g_{0}} d r\right\}
$$

and

$$
\delta p(r)=-\frac{\delta m}{4 \pi r^{3}} \frac{1+8 \pi p_{0} r^{2}}{1-2 m_{0} / r}
$$

Here $\delta \rho_{c}$ is the shift in the central density. By explicitly combining these formulae we have

$$
\begin{gathered}
\delta p(r)=\frac{\delta p_{c}}{\left[1+r g_{0}\right]^{2}} \frac{1+8 \pi p_{0} r^{2}}{1-\frac{2 m_{0}}{r}} \\
* \exp \left\{2 \int_{0}^{r} g_{0} \frac{1-r g_{0}}{1+r g_{0}} d r\right\},
\end{gathered}
$$

and

$$
\delta \rho(r)=-\frac{1}{r^{2}} \frac{d}{d r}\left(\frac{\delta p(r) r^{3}}{1+2 r g_{0}(r)}\right)
$$

\section{Charged Fluid Spheres}

\subsection{Anisotropic Fluid Spheres and Generalized TOV Equation}

The perfect fluid sphere possesses key properties such as the isotropy of pressure with no viscosity, shear stresses, or heat conduction. Using perfect fluid spheres, stars in our universe can be modeled. If charges are added to a perfect fluid sphere, the latter becomes anisotropic. An anisotropic sphere can also be made by a scalar field. Perfect fluid spheres are the first approximation of solution for many objects. However, there are also many other spherical objects that do not fit the properties of perfect fluid spheres. One kind of such object is a neutron star. The radial pressure of the stars differs from the transverse pressure. The applied idea for these stars is referred to as anisotropic fluid spheres.

$$
T_{\hat{a} \hat{b}}=\left[\begin{array}{cccc}
\rho & 0 & 0 & 0 \\
0 & p_{r} & 0 & 0 \\
0 & 0 & p_{t} & 0 \\
0 & 0 & 0 & p_{t}
\end{array}\right] .
$$

We define new solution of charged fluid spheres as the metric of spacetime

$$
d s^{2}=-\zeta_{0}(r)^{2} d t^{2}+\frac{d r^{2}}{B_{0}(r)}+r^{2} d \Omega^{2}
$$

or with the notation $\left\{\zeta_{0}, B_{0}\right\}$ by setting

$$
G_{\hat{r} \hat{r}}-G_{\hat{\theta} \hat{\theta}}=8 \pi \Delta,
$$

where $\Delta$ is an arbitrary function in terms of radius $r$. The Einstein's tensors were written in a non-coordinate form, which represents an observer's view of physical quantities.

\subsection{Modeling Static Anisotropy}

For describing the inertia properties of anisotropy, we need 
to modify the new energy momentum stress tensor for anisotropy. It can be modeled in terms of a linear combination of the "perfect fluid", "electromagnetic field", and "massless scalar field",

From the definition [10], the tensor for perfect fluid spheres is

$$
T_{f}^{a b}=\left(\rho_{f}+p_{f}\right) V^{a} V^{b}+p_{f} g^{a b} .
$$

The tensor for electromagnetic field is

$$
T_{e m}^{a b}=F^{a c} g_{c d} F^{b d}-\frac{1}{4} g^{a b}\left(F_{c d} F^{c d}\right) .
$$

The tensor for (minimally coupled) massless scalar field is

$$
T_{s}^{a b}=\phi^{; a} \phi^{; b}-\frac{1}{2} g^{a b}\left(g^{c d} \phi_{; c} \phi_{; d}\right) .
$$

Using the covariant conservation of total stress energy, $T^{a b}{ }_{; b}=0$, we obtain a relation of perfect fluid density with pressure, scalar field, and the field strength tensor of the electromagnetic field

$$
\left(\rho_{f}+p_{f}\right) V_{; b}^{a} V^{b}+g^{a b}\left(\left[p_{f}\right]_{; b}+\sigma_{s} \phi_{; b}\right)-F^{a b}\left(\sigma_{e m} V_{b}\right)=0,
$$

After we obtain the equation of anisotropy in terms of pressure and density, we have to assume that the equation must be equivalent to the TOV equation.

This is the modified TOV equation [10]

$$
\begin{gathered}
\frac{d p_{f}}{d r}=-\frac{\left[\rho_{f}+p_{f}\right]\left[m(r)+4 \pi p_{f} r^{3}\right]}{r^{2}\left[1-\frac{2 m(r)}{r}\right]} \\
-\frac{\sigma_{e m} E}{\sqrt{1-\frac{2 m(r)}{r}}}-\sigma_{s} \frac{d \phi}{d r}, \\
\frac{d m}{d r}=4 \pi \rho r^{2}=4 \pi\left(\rho_{f}+\rho_{e m}+\rho_{s}\right) r^{2},
\end{gathered}
$$

where $\rho_{f}, \rho_{e m}, \rho_{s}, \sigma_{e m}, \sigma_{s}$, and $\phi$ represent perfect fluid density, electromagnetic density, electromagnetic charge, and the massless scalar charge and field, respectively.

\section{The Effect of Charge on Pressure}

\subsection{Special Case: When $\rho$ Is Constant and $\sigma_{s}$ Is Zero}

In this case, the generalized TOV equation becomes

$$
\frac{d p_{f}}{d r}=-\frac{\left[\rho_{f}+p_{f}\right]\left[m(r)+4 \pi p_{f} r^{3}\right]}{r^{2}\left[1-\frac{2 m(r)}{r}\right]}-\frac{\sigma_{e m} E}{\sqrt{1-\frac{2 m(r)}{r}}}
$$

supplemented with

$$
\frac{d m}{d r}=4 \pi \rho r^{2}=4 \pi\left(\rho_{f}+\rho_{e m}\right) r^{2} .
$$

Integrating the above equation, we obtain

$$
m(r)=\frac{4}{3} \pi \rho r^{3}
$$

Substituting $m(r)$ into the generalized TOV equation, we can numerically solve for $p_{f}$ as shown in Figure 2.

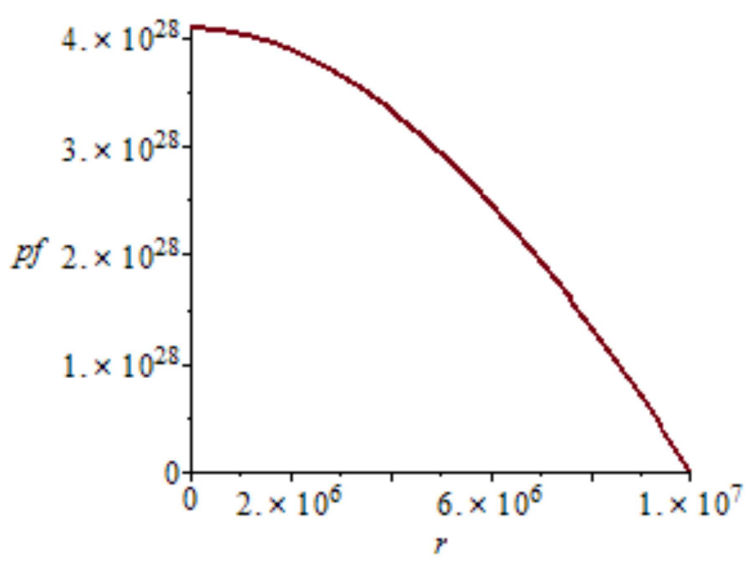

Figure 1. The fluid pressure as a function of radius.

\subsection{The Effect Between Charge and Pressure}

In this part, we will investigate how the charge affects the pressure in an anisotropic fluid sphere. From equation (11), we set the mass, density, and electric field fixed, and vary the charge density in order to examine how the pressure changes. The effect of charge on pressure is shown in the Table 1.

Table 1. This table shows the effect of charged density on pressure for generalized TOV equation in special case ( $\rho=$ constant).

\begin{tabular}{ll}
\hline Charge density $\left(\mathbf{C} / \mathbf{m}^{3}\right)$ & Pressure $\left(\mathbf{N} / \mathbf{m}^{2}\right)$ \\
\hline 1 & $4.08 \times 10^{28}$ \\
$2 \times 10^{11}$ & $7.25 \times 10^{29}$ \\
$3 \times 10^{11}$ & $1.08 \times 10^{30}$ \\
$4 \times 10^{11}$ & $2.00 \times 10^{30}$ \\
$5 \times 10^{11}$ & $1.84 \times 10^{30}$ \\
\hline
\end{tabular}

From the table, we can see that when the charge density increases, the pressure also increases. The charge causes the pressure to increase. Anisotropic fluid sphere has a pressure greater than that of perfect fluid sphere for equal mass, density, and electric field. For example, we compare the case of $\sigma_{e m}=10^{11} \mathrm{~kg} / \mathrm{m}^{3}$ with the case of $\sigma_{e m}=5 \times$ $10^{11} \mathrm{~kg} / \mathrm{m}^{3}$ as shown in Figures 2 and 3, respectively.

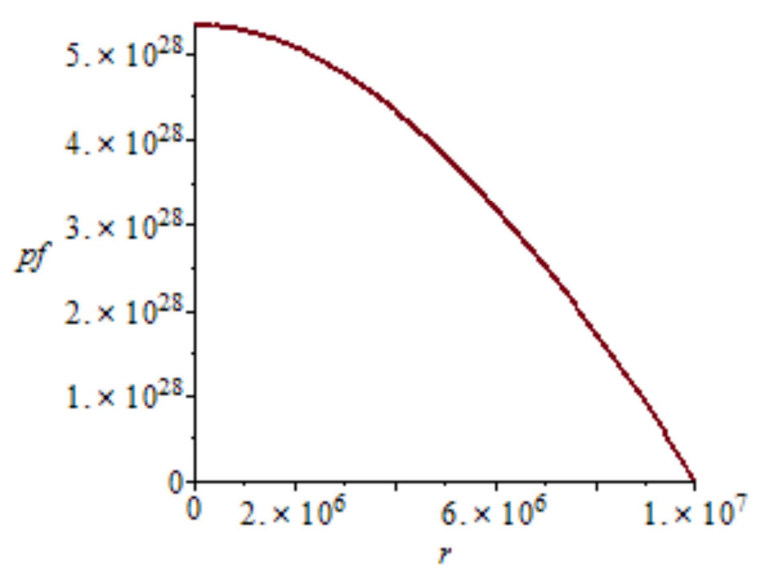

Figure 2. The fluid pressure for $\sigma_{e m}=10^{11} \mathrm{~kg} / \mathrm{m}^{3}$ 


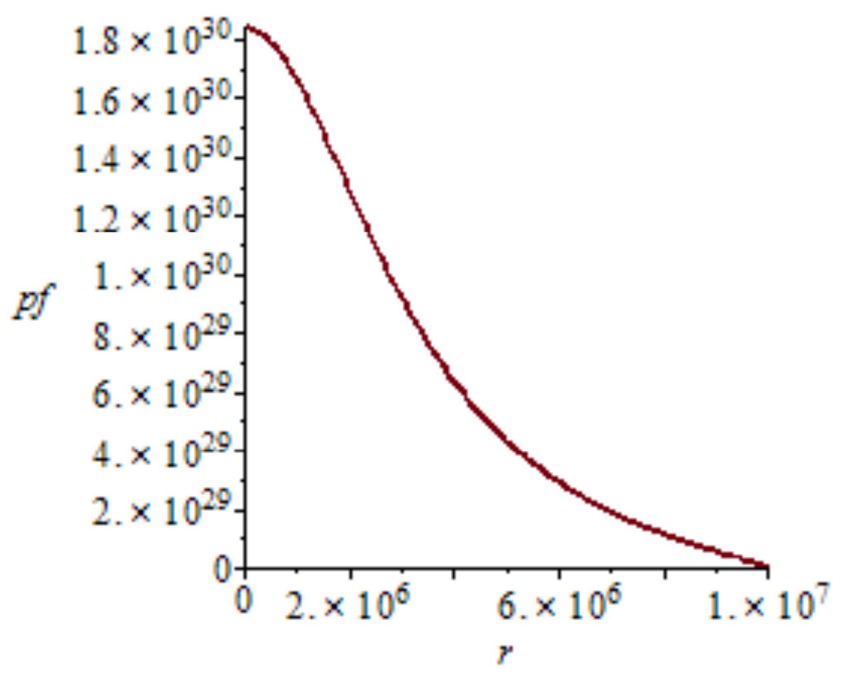

Figure 3. The fluid pressure for $\sigma_{e m}=5 \times 10^{11} \mathrm{~kg} / \mathrm{m}^{3}$.

From these graphs, we can see that when the charge is denser, the fluid pressure increases. In this case, when the charge density increases by 5 times, the pressure can increase by a 100 times. Moreover, the effect of fluid density on pressure is also investigated, as shown in Table 2.

Table 2. This table shows the effect of fluid density on pressure for generalized TOV equation in special case ( $\rho=$ constant).

\begin{tabular}{ll}
\hline Density $\left(\mathrm{kg} / \mathrm{m}^{3}\right)$ & Pressure $\left(\mathrm{N} / \mathbf{m}^{2}\right)$ \\
\hline $1 \times 10^{12}$ & $4.08 \times 10^{28}$ \\
$1.1 \times 10^{12}$ & $6.28 \times 10^{28}$ \\
$1.2 \times 10^{12}$ & $1.04 \times 10^{29}$ \\
$1.3 \times 10^{12}$ & $2.07 \times 10^{29}$ \\
$1.4 \times 10^{12}$ & $9.47 \times 10^{29}$ \\
\hline
\end{tabular}

Similarly, when the fluid density increases, the pressure also increases. This means that the denser charged stars have a pressure greater than the less compact stars.

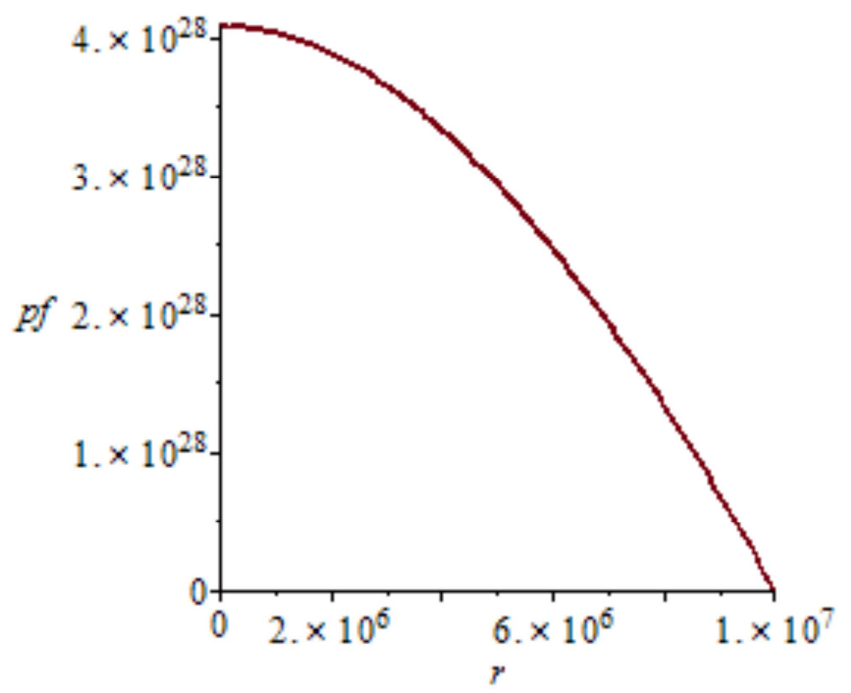

Figure 4. The fluid pressure for $\rho_{f}=10^{22} \mathrm{~kg} / \mathrm{m}^{3}$.

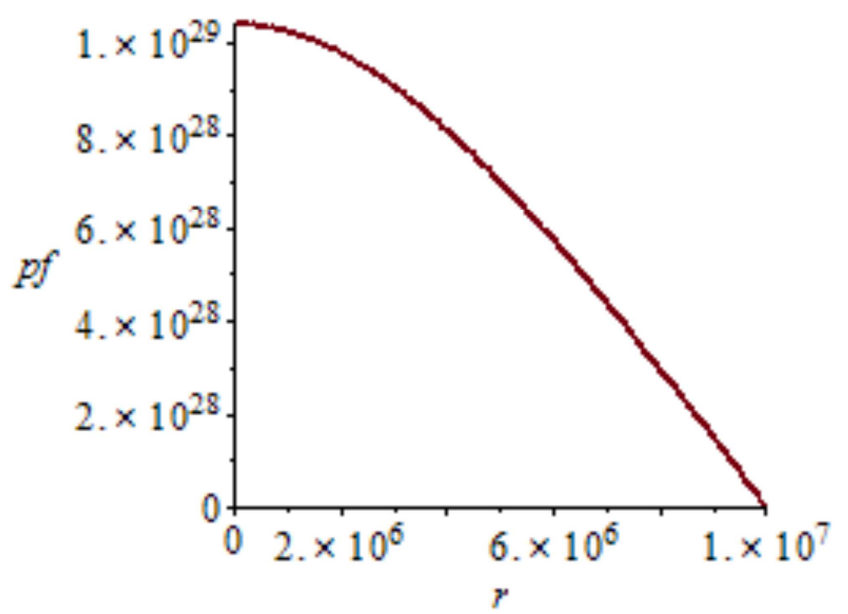

Figure 5. The fluid pressure for $\rho_{f}=1.2 \times 10^{22} \mathrm{~kg} / \mathrm{m}^{3}$.

Figures 4 and 5 show the effect of fluid density on pressure. We can see that when the fluid density increases by 1.2 times, the pressure can increase by approximately 100 times. Moreover, when the radius decreases, the pressure increases. That is the center of star has the highest pressure.

\section{The Transmission and Reflection Probabilities for Tolman-Bayin Type of Anisotropic Fluid Sphere}

\subsection{The Transmission Probability}

Referring to P. Boonserm, and M Visser [23], we use a similar concept to find the transmission and reflection probabilities for the Tolman-Bayin type of charged fluid sphere.

The wave that can be observed by an observer away from a black hole is the only transmitted wave. The incident wave is a blackbody radiation because a black hole is a blackbody. However, the transmitted wave is no longer a blackbody radiation due to the modification from the curvature of spacetime. We call the transmitted wave as a greybody radiation. The transmission probability is called the greybody factor for black hole systems. The greybody factor is a quantity containing information about the percentage of Hawking radiation that can reach infinity. In addition, we can derive the greybody factor by solving the Schrodinger-like equation. However, we cannot, in general, find the exact solutions [23-25].

For the static, spherically symmetric background, the metric takes the form $[10,26]$

$$
d s^{2}=\zeta_{0}(r)^{2} d t^{2}-\frac{d r^{2}}{B_{0}(r)}-r^{2} d \Omega^{2} .
$$

Substituting in the Einstein's field equation and performing some manipulation, we obtain [26]

$$
\frac{d C}{d r}=\left(\frac{\sqrt{B_{0}}}{r^{2}} \frac{d}{d r}\left(\frac{1}{\sqrt{B_{0}}}\right)-\frac{\frac{1}{B_{0}}-1}{r^{3}}\right)+\sqrt{B_{0}} \frac{d}{d r}\left(\frac{1}{\sqrt{B_{0}}}\right) C-C^{2} r+\frac{2 q^{2}}{B_{0} r^{5}},
$$


where

$$
C=\frac{\zeta_{0^{\prime}}(r)}{r \zeta_{0}(r)} .
$$

Matching this interior solution with the exterior Reissner-Nordstrom at $\mathrm{r}=\mathrm{a}$, we assume the total charge to be

$$
q(a)=K a^{n},
$$

where $\mathrm{K}$ is constant. Moreover, we can find total gravitational mass

$$
m=\frac{n a^{2}(2-n)+2 q^{2}}{2\left(1+2 n-n^{2}\right) a} .
$$

For simplicity, we are interested in the case $\mathrm{n}=0$. So we obtain

$$
\begin{gathered}
\zeta_{0}^{2}=1-\frac{2 m}{a}+\frac{q^{2}}{a^{2}} . \\
B_{0}=1-\frac{2 m}{a}+\frac{q^{2}}{a^{2}}=1-\frac{2 q^{2}}{a^{2}} .
\end{gathered}
$$

In the presence of a scalar field, it is possible for anisotropic fluid sphere to radiate scalar waves. Therefore, we can find the transmission probability of the scalar waves in propagating to a distant place, or spatial infinity. In [24, 27], the rigorous bound on the transmission probability was calculated for the Myers-Perry black hole. In this paper, we study the Tolman-Bayin type of charged fluid sphere, which takes the form [26]

$$
d s^{2}=\left(1-\frac{2 m}{a}+\frac{q^{2}}{a^{2}}\right)^{2} d t^{2}-\left(1+\frac{q^{2}}{a^{2}}\right)^{-2} d r^{2}-r^{2} d \Omega^{2} .
$$

The rigorous bound on the transmission probability is given by [24-25]

$$
T \geq \operatorname{sech}^{2}\left[\frac{1}{2 \omega} \int_{-\infty}^{\infty} V_{l}(r) d r_{*}\right] .
$$

For the Tolman-Bayin type of charged fluid sphere, the potential takes the form

$$
V(r)=\frac{l(l+1)}{r^{2}}\left(1-\frac{2 m}{a}+\frac{q^{2}}{a^{2}}\right)^{2}
$$

and the tortoise coordinate is given by

$$
\frac{d r_{*}}{d r}=\frac{1}{\left(1-\frac{2 m}{a}+\frac{q^{2}}{a^{2}}\right)\left(1-\frac{2 q^{2}}{a^{2}}\right)} .
$$

Therefore, the rigorous bounds on the transmission probability is

$$
\begin{aligned}
T \geq & \operatorname{sech}^{2}\left[\frac{1}{2 \omega} \int_{-\infty}^{\infty} \frac{l(l+1)}{r^{2}}\left(1-\frac{2 m}{a}+\frac{q^{2}}{a^{2}}\right)^{2} d r_{*}\right] \\
& =\operatorname{sech}^{2}\left[\frac{1}{2 \omega} \int_{r_{0}}^{\infty} \frac{l(l+1)}{r^{2}} \frac{1-\frac{2 m}{a}+\frac{q^{2}}{a^{2}}}{1-\frac{2 q^{2}}{a^{2}}} d r\right]
\end{aligned}
$$

$$
\begin{aligned}
& =\operatorname{sech}^{2}\left[\frac{1}{2 \omega} \int_{r_{0}}^{\infty} \frac{l(l+1)}{r^{2}} \frac{a^{2}-2 m a+q^{2}}{a^{2}-2 q^{2}} d r\right] \\
& =\operatorname{sech}^{2}\left[\frac{1}{2 \omega} l(l+1) \frac{a^{2}-2 m a+q^{2}}{a^{2}-2 q^{2}}\left(-\frac{1}{r}\right)_{r_{0}}^{\infty}\right] \\
& =\operatorname{sech}^{2}\left[\frac{1}{2 \omega} l(l+1) \frac{a^{2}-2 m a+q^{2}}{a^{2}-2 q^{2}}\left(\frac{1}{r_{0}}\right)\right] .
\end{aligned}
$$

The rigorous bound on the transmission probability is plotted with $\omega$ as shown in Figure 6.

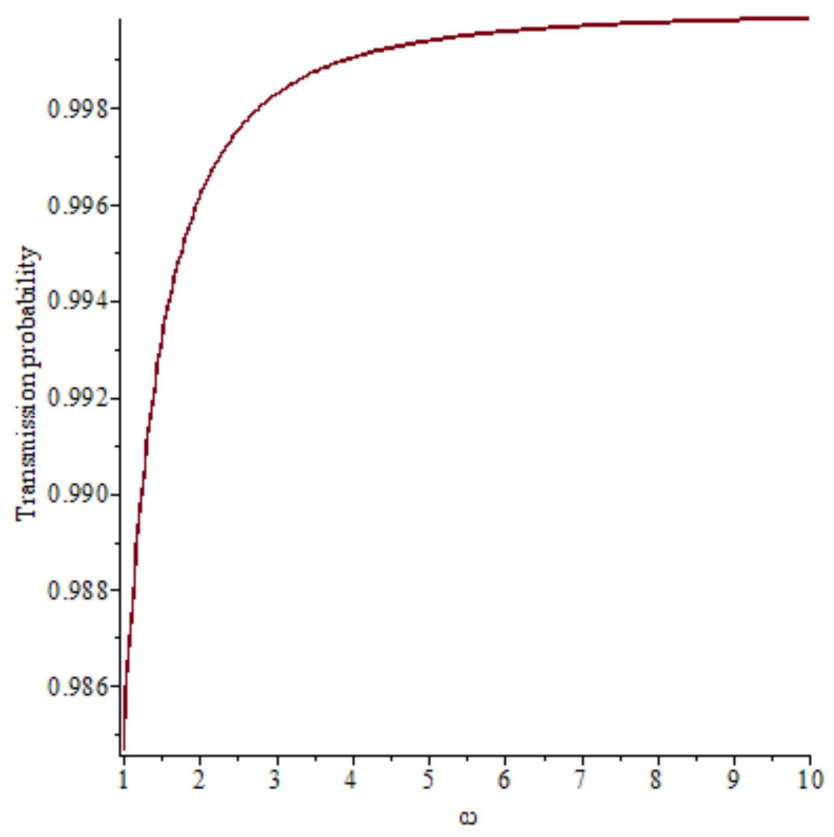

Figure 6. The rigorous bound on the transmission probability as a function of $\omega$.

From the graph, we can see that the rigorous bound on the transmission probability increases as the wave's energy increases. This means that the waves with higher energy can penetrate to spatial infinity with a higher probability than lower energy waves.

\subsection{The Reflection Probability}

From the law of conservation, the relationship between the reflection probability and the transmission probability satisfies [25]

$$
R+T=1 .
$$

Therefore, for the given transmission probability, the reflection probability can be obtained

$$
R=1-T=1-\operatorname{sech}^{2}\left[\frac{1}{2 \omega} l(l+1) \frac{a^{2}-2 m a+q^{2}}{a^{2}-2 q^{2}}\left(\frac{1}{r_{0}}\right)\right] .
$$

The rigorous bound on the reflection probability is plotted with $\omega$ as shown in Figure 7. 


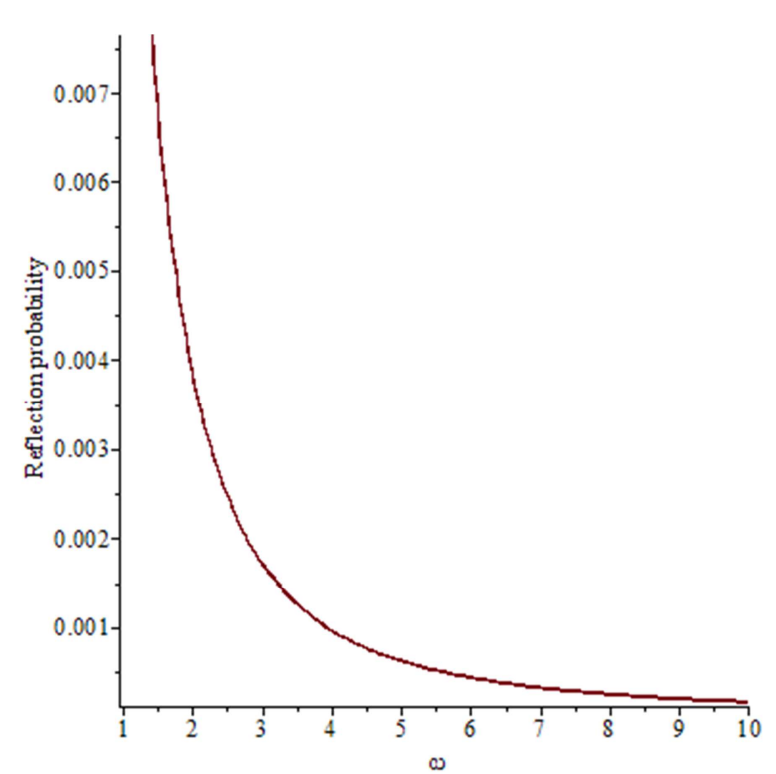

Figure 7. The rigorous bound on the reflection probability as a function of $\omega$.

From Figure 7, we can see that the rigorous bound on reflection probability decreases with increasing wave energy. This relationship is an inverse of the relationship associated with the transmission probability. That is, the increase in the rigorous bound on reflection probability corresponds to the decrease in the rigorous bound on transmission probability, satisfying the law of conservation within the equation (26).

\section{Conclusion}

In this paper, we derived the generalized TOV equation for anisotropic fluid sphere. We investigated the effect of charge on pressure in the absence of a scalar field, and with a constant charge density. The result shows that the presence of charge can add to the pressure of charged stars. Moreover, a denser star has more pressure than the less compact stars. Finally, we calculated the rigorous bound on the transmission probability and the reflection probability for the Tolman-Bayin type of charged fluid sphere. We found that high energy waves have a higher probability in reaching spatial infinity than low energy waves.

For future work, spherical objects in reality can be more easily observed in terms of pressure and density. Therefore, building a solution generating theorem in terms of pressure and density allows us to gain a clear understanding of star-like object. In this work, the solution generating theorem algorithm is applied to the generalized TOV equation. We can find new solutions from the theorems. Moreover, the type of solutions can also be classified by the theorems. We can also see the interrelationship between the anisotropic solutions.

\section{Acknowledgements}

This project was funded by the Ratchadapisek Sompoch Endowment Fund, Chulalongkorn University (Sci-Super 2014-032), by a grant for the professional development of new academic staff from the Ratchadapisek Somphot Fund at
Chulalongkorn University, by the Thailand Research Fund (TRF), and by the Office of the Higher Education Commission (OHEC), Faculty of Science, Chulalongkorn University. PB was additionally supported by a scholarship from the Royal Government of Thailand. TN was also additionally supported by a scholarship from the Development and Promotion of Science and Technology talent project (DPST). NJ was also additional supported by a travel grant from Department of Physics, Faculty of Science, Chulalongkorn University.

\section{References}

[1] M. S. R. Delgaty and K. Lake, "Physical acceptability of isolated, static, spherically symmetric, perfect fluid solutions of Einstein's equations", Compute. Phys. Commun. 115 (1998) 395 [arXiv: gr-qc/9809013].

[2] S. Rahman and M. Visser, "Spacetime geometry of static fluid spheres", Class. Quant. Grav. 199352002 [arXiv: gr-qc/0103065].

[3] P. Boonserm, M. Visser and S. Weinfurtner, "Generating perfect fluid spheres in general relativity", Phys. Rev. D 71 (2005) 124037 [arXiv: gr-qc/0503007].

[4] P. Boonserm, M. Visser and S. Weinfurtner, "Solution generating theorems for the TOV equation", Phys. Rev. D 76 (2007) 044024 [arXiv: gr-qc/0607001].

[5] D. Martin and M. Visser, "Bounds on the interior geometry and pressure profile of static fluid spheres," Class. Quant. Grav. 20 (2003) 3699-3716 [arXiv: gr-qc/ 0306038].

[6] K. Lake, "All static spherically symmetric perfect fluid solutions of Einstein's Equations," Phys. Rev. D 67 (2003) 104015 [arXiv: gr-qc/0209104].

[7] D. Martin and M. Visser, "Algorithmic construction of static perfect fluid spheres," Phys. Rev. D 69 (2004) 104028 [arXiv: gr-qc/0306109].

[8] S. Ray, A. L. Esp'indola, M. Malheiro, J. P. S. Lemos, and V. T. Zanchin, "Electrically charged compact stars and formation of charged black holes", Phys. Rev. D 68 084004, 2003 [arXiv: astro-ph/0307262].

[9] W. Barreto, L. Castillo, and E. Barrios, "Central equation of state in spherical characteristic evolutions", Phys. Rev. D 80 0840072009 [arXiv: 0909.4500 [gr-qc]].

[10] P. Boonserm, T. Ngampitipan and M. Visser, "Mimicking static anisotropic fluid spheres in general relativity", International Journal of Modern Physics D (2015): 1650019 [arXiv: $1501.07044 \mathrm{v} 3$ [gr-qc]].

[11] F. Siebel, J. A. Font, and P. Papadopoulos, "Scalar field induced oscillations of neutron stars and gravitational collapse", Phys. Rev. D 650240212001 [arXiv: gr-qc/0108006].

[12] K. Schwarzschild, "On the gravitational field of a sphere of incompressible fluid according to Einstein's theory", Sitzungsber. Preuss. Akad. Wiss. Berlin (Math. Phys.) 1916 (1916) 424 [arXiv: physics/9912033 [physics.hist-ph]].

[13] S. Carroll, "Spacetime and geometry: an introduction to general relativity", Pearson new international edition, U.S.A., Pearson Education Limited, 2014. 
[14] H. Bondi, "Spherically symmetrical models in general relativity”, Mon. Not. Roy. Astron. Soc. 107 (1947) 410.

[15] H. A. Buchdahl, "General relativistic fluid spheres", Phys. Rev. 116 (1959) 1027-1034.

[16] A. Sulaksono, "Anisotropic pressure and hyperon in neutron stars," Int. J. Mod. Phys. E 24 (2015) 01, 1550007 [arXiv: 1412.7274 [nucl-tn]].

[17] P. Boonserm, "Some exact solution in general relativity", MSc. Thesis, Victoria University of Wellington, 2006.

[18] D Kramer, H Stephani, E Herlt, and M MacCallum, "Exact solutions of Einstein's field equations", (Cambridge University Press, England, 1980).

[19] S. Ray, A. L. Esp'indola, M. Malheiro, J. P. S. Lemos, and V. T. Zanchin, "Electrically charged compact stars and formation of charged black holes", Phys. Rev. D 68 084004, 2003 [arXiv: astro-ph/0307262]

[20] W. Barreto, L. Castillo, and E. Barrios, "Central equation of state in spherical characteristic evolutions", Phys. Rev. D 80 0840072009 [arXiv: 0909.4500 [gr-qc]].

[21] L. Herrera, J. Ospino and A. Di Prisco, "All static spherically symmetric anisotropic solutions of Einstein's equation", Phys. Rev. D 77 (2008) 027502 [arXiv: 0712.0713 [gr-qc]].
[22] Chandrasekhar, S. (1998). The Mathematical Theory of Black Holes (Reprinted ed.). Oxford University Press. p. 205. ISBN 0-19850370-9. Retrieved 13 May 2013.

[23] P. Boonserm, and M. Visser, "Bounding the greybody factors for Schwarzschild black holes.” Phys. Rev. D 78(10) 101502 2008 .

[24] T. Ngampitipan, "Rigurous bounds on grey body factors for various types of black holes", $\mathrm{Ph}$. D. Thesis, Chulalongkorn University, 2014.

[25] P. Boonserm, "Rigorous Bounds on Transmission, Reflection, and Bogoliubov Coefficients", Ph. D. Thesis, Victoria University of Wellington (2009) [arXiv: 0907.0045 [mathph]].

[26] Ray, Saibal, and Basanti Das, "Tolman-Bayin type static charged fluid spheres in general relativity." Monthly Notices of the Royal Astronomical Society 349.4 (2004), 1331-1334.

[27] P. Boonserm, A. Chatrabhuti, T. Ngampitipan, and M. Visser, "Greybody factors for Myers-Perry black holes", J. Math. Phys. 55, 112502 (2014) [arXiv: 1405.5678[gr-qc]].

[28] K. Komathiraj ans S. D. Maharaj, "A class of charged relativistic spheres", Mathematical and Computational Applications 15, 665-673, 2010. 